\title{
BMJ Open Healthcare resource consumption for intermittent urinary catheterisation: cost-effectiveness of hydrophilic catheters and budget impact analyses
}

\author{
Carla Rognoni, ${ }^{1}$ Rosanna Tarricone ${ }^{1,2}$
}

To cite: Rognoni C, Tarricone R. Healthcare resource consumption for intermittent urinary catheterisation: costeffectiveness of hydrophilic catheters and budget impact analyses. BMJ Open 2017;7: e012360. doi:10.1136/ bmjopen-2016-012360

- Prepublication history and additional material is available. To view please visit the journal (http://dx.doi.org/ 10.1136/bmjopen-2016012360).

Received 19 April 2016 Revised 5 September 2016 Accepted 18 October 2016

\footnotetext{
${ }^{1}$ Centre for Research on Health and Social Care Management (CERGAS), Bocconi University, Milan, Italy

2Department of Policy Analysis and Public Management, Bocconi University, Milan, Italy

Correspondence to Dr Carla Rognoni; carla.rognoni@unibocconi.it
}

\section{ABSTRACT}

Objectives: This study presents a cost-effectiveness analysis comparing hydrophilic coated to uncoated catheters for patients performing urinary intermittent catheterisation. A national budget impact analysis is also included to evaluate the impact of intermittent catheterisation for management of bladder dysfunctions over a period of 5 years.

Design: A Markov model (lifetime horizon, 1 year cycle length) was developed to project health outcomes (life years and quality-adjusted life years) and economic consequences related to patients using hydrophilic coated or uncoated catheters. The model was populated with catheter-related clinical efficacy data retrieved from randomised controlled trials and quality-of-life data (utility weights) from the literature. Cost data (EUR, 2015) were estimated on the basis of healthcare resource consumption derived from an e-survey addressed to key opinion leaders in the field.

Setting: Italian Healthcare Service perspective. Population: Patients with spinal cord injury performing intermittent urinary catheterisation in the home setting.

Main outcome measures: Incremental costeffectiveness and cost-utility ratios (ICER and ICUR) of hydrophilic coated versus uncoated catheters and associated healthcare budget impact.

Results: The base-case ICER and ICUR associated with hydrophilic coated catheters were $€ 20761$ and $€ 24$ 405, respectively. This implies that hydrophilic coated catheters are likely to be cost-effective in comparison to uncoated ones, as proposed Italian threshold values range between $€ 25000$ and $€ 66400$. Considering a market share at year 5 of $89 \%$ hydrophilic catheters and $11 \%$ uncoated catheters, the additional cost for Italy is approximately $€ 12$ million in the next 5 years (current market share scenario for year 0: $80 \%$ hydrophilic catheters and $20 \%$ uncoated catheters).

Conclusions: Considered over a lifetime, hydrophilic coated catheters are potentially a cost-effective choice in comparison to uncoated ones. These findings can assist policymakers in evaluating intermittent catheterisation in patients with spinal cord injury.

\section{Strengths and limitations of this study}

- This paper presents a cost-effectiveness analysis comparing hydrophilic coated to uncoated catheters in spinal cord-injured patients performing intermittent catheterisation. Healthcare resource consumption was derived from an e-survey addressed to key opinion leaders to provide realworld data.

- The study combines a cost-effectiveness analysis with a budget impact analysis. The addition of the budget impact analysis gives further evidence as to the overall impact of adopting the device for decision-makers to review.

- Data derived from self-reported questionnaires may be limited by varying recollection and poor generalisability. Variables derived from prospective observational multi-centre studies would increase the validity of the current model.

- The findings of this study support the use of hydrophilic coated catheters but are limited to costs from a healthcare perspective. A broader evaluation, also including costs from a societal perspective, would increase the understanding of the economic sustainability of these devices.

\section{INTRODUCTION}

Injuries to the spinal cord (SCI) affect bladder functionality and cause motor or sensory deficits of a diverse nature and extent. Many of these conditions affect bladder functionality and cause what is known as a neurogenic bladder, often characterised by voiding problems. This clinical condition has a negative impact on health-related quality of life, and the associated economic costs can be overwhelming for patients already hampered with neurological problems. Healthcare usage may be excessive for these patients, including emergency department visits and subsequent hospitalisations. ${ }^{1}$

In the community setting, the management of a neurogenic bladder frequently 
involves intermittent catheterisation (IC). With this technique, a catheter is temporarily used to remove urine from the bladder. As neurogenic bladder is often a permanent condition, IC may be required for a long period of time, often several times a day. There are different catheters available for IC, for example, disposable catheters with a hydrophilic polymer surface coating, disposable catheters with pre-packaged water based lubricant (gel reservoir) and uncoated catheters. While there is a lack of strong evidence demonstrating the effectiveness of any particular catheter design, technique or strategy, ${ }^{2}$ the use of different kinds of catheters in the community may have different economic consequences. To the best of our knowledge, two cost-effectiveness studies ${ }^{3}{ }^{4}$ have compared lifetime quality-adjusted life years (QALYs) and costs of different types of catheter from the UK perspective. Although both studies focused on the management of urinary tract infections (UTIs), the first ${ }^{3}$ based its analysis on the annual probability of experiencing at least one UTI for the different catheters considered (without taking into account the mean number of UTIs experienced in the same time by the patients' cohort) and their short-term consequences. The second study ${ }^{4}$ focused on the average UTI rate per patient and month for hydrophilic coated and uncoated catheters and considered long-term sequelae such as kidney impairment. Considering a lifetime horizon, the study by Clark $e t a l^{t}$ showed that hydrophilic coated catheters are costeffective when compared to uncoated catheters. Conversely, the study by Bermingham et al reported that the reuse and cleansing of uncoated catheters is the most cost-effective alternative in comparison to all other catheter types. It should however be noted that reuse and cleaning of uncoated catheters may be regarded as an off-label procedure not supported by all regulating bodies. The divergent results from previous costeffectiveness studies confirm that assumptions made, and the manner in which clinical data are chosen, highly affect the model construction and conclusions from the analysis, even in the same country setting.

One of the major advantages of IC is the significant reduction in the risk of catheter-associated UTIs, ensuring urinary tract health in general and preservation of kidney function in particular. ${ }^{5}$ Despite the efforts in reducing the risk of UTIs, they still cause high morbidity and frequent hospitalisations for people with neurogenic bladder. Repeated cycles of antibiotic therapy in patients with recurrent UTIs also contribute to 'antibiotic resistance, ${ }^{7}$ which in turn increases the need for new effective treatment options. For these reasons, UTIs entail a significant economic burden for patients, their families and healthcare systems. ${ }^{8}$

Studies that attempted to estimate the burden of UTIs from the healthcare system perspective report costs ranging from $€ 523$ to $€ 4167,{ }^{9-14}$ with more complicated UTIs likely to be associated with higher costs. The high variability in costs relates to several aspects. For example, UTI definition (bacteriuria vs symptomatic UTI), study setting (hospital vs community), study population (general patients in hospital vs specific populations) and cost definitions can vary. The latter may consider direct healthcare costs only (eg, medications, therapies), or include indirect costs to society as productivity losses. The use of different payer perspectives (society and/or healthcare system) may also result in different UTI cost values.

In addition to the risk of UTI, IC performed several times a day poses a risk for urethral trauma. Urethral trauma can occur with or without the presence of haematuria and is associated with an increased risk of UTI. ${ }^{15}{ }^{16}$ Damage to the urethra is less likely to occur with a lubricated catheter. ${ }^{17}$

A catheter reducing the risks of urethral trauma and/ or UTI may limit the economic burden for the healthcare system and may increase the quality of life for patients. A cost-effectiveness analysis (CEA) permits a systematic evaluation of the costs and quality-of-life consequences of different treatment regimens, highlighting the option that would have the highest net benefit.

The aim of this study was to perform a CEA from an Italian Healthcare Service perspective, comparing the two catheter types most frequently used for IC (ie, disposable hydrophilic coated or uncoated plastic catheters). This was done to add value to previously conflicting results of CEAs evaluating different catheter types, and to identify the most cost-effective catheter alternative for the Italian setting. A budget impact analysis (BIA) was also conducted to evaluate the impact on the Italian healthcare budget of IC for the management of bladder dysfunctions over a period of 5 years.

\section{METHODS}

The clinical effectiveness of each catheter was retrieved from randomised controlled trials (RCTs) focusing on the community perspective that were published in the literature. Cost data were estimated on the basis of diagnosis-specific healthcare resource usage, derived from an e-survey addressed to key opinion leaders in the field. Since clinical data were mainly reported for SCI patients, the model considered these as an applicable study population. The study focused mainly on UTIs and episodes of haematuria, as the former are the most frequent complications in patients performing IC, while the latter occur regularly in one-third of patients on a long-term basis. ${ }^{18}$

\section{Systematic literature review and clinical data synthesis}

A systematic literature review was performed in June 2016 to retrieve RCTs, comparing hydrophilic coated and uncoated catheters for IC and reporting outcomes on UTIs and haematuria. A systematic search was conducted on PubMed, Embase, the Cochrane Library and Web of Science databases to retrieve clinical evidence (see online supplementary appendix for detailed search strategy and PRISMA statement). 
In Italy, single-use catheters are considered the standard method for IC, and four catheters per day are delivered to users by local health agencies. ${ }^{19}$ Reuse of catheters is not present or relevant to the Italian healthcare system, so clinical evidence considering catheter reuse was discarded. Studies not reporting UTI frequencies per patient were also excluded. The studies by Cardenas $e t a l^{20}{ }^{21}$ and Sarica $e t a l^{22}$ focused on SCI patients and reported data useful for the analysis. Data reported by Clark et at derived from an internal report of the study conducted by De Ridder $e t a t^{23}$ were also included.

Table 1 reports UTI rates according to the methods presented in Clark et al, ${ }^{4}$ distinguishing the following settings: hospital period, community setting and combined scenario (hospital and community settings).

For haematuria, three studies ${ }^{21-23}$ reporting useful data were identified by the systematic literature search (table 2).

\section{The model}

As the management of patients performing IC is an evolving process, Markov multistate models were chosen for the health economic evaluation. A decision tree, combined with two Markov models, was designed to project lifetime health outcomes (life years and QALYs) and economic consequences related to SCI patients performing IC with hydrophilic or non-hydrophilic urinary catheters.

The Markov model (figure 1) includes the following health states: Alive, Symptomatic UTI, Haematuria and Death. A symptomatic UTI can either resolve or become an antibiotic-resistant UTI. In this case, the model distinguishes between first-line-resistant UTI, multidrugresistant UTI and bacteraemia. Multidrug-resistant UTI and bacteraemia represent severe UTIs that can eventually cause patient death.

It is acknowledged that complications other than the ones included in the model health states may be relevant for patients practicing IC. For example, other infections and inflammations such as epididymo-orchitis, urethritis and prostatitis may occur as a complication of IC as well as strictures, false passage and bladder stones. ${ }^{24}$ The 'Alive' state accounts for baseline rates of these kinds of complications, which have been elicited by key opinion leaders in the field and assumed equal for hydrophilic coated and uncoated catheters (see details in the Results section-Healthcare resource consumption and costs).

A hypothetical cohort of 40-year-old, $80 \%$ male patients enters the Markov process in the 'Alive' state. Population characteristics are assumed to be similar to those previously reported for SCI patients performing IC in Italy. ${ }^{25}$

\begin{tabular}{|c|c|c|c|c|c|}
\hline Study & Patients & $\begin{array}{l}\text { Number of } \\
\text { events }\end{array}$ & $\begin{array}{l}\text { Rate per patient } \\
\text { per month }\end{array}$ & Weighted mean & Rate ratio \\
\hline \multicolumn{6}{|l|}{ Hospital period } \\
\hline \multicolumn{6}{|l|}{ Uncoated } \\
\hline Cardenas $^{21}$ & 114 & & 0.68 & 0.61 & 0.78 \\
\hline De Ridder ${ }^{23}$ & 61 & & 0.55 & & \\
\hline Sarica $^{22}$ & 10 & 4 & 0.27 & & \\
\hline \multicolumn{6}{|l|}{ Hydrophilic } \\
\hline Cardenas $^{21}$ & 105 & & 0.54 & 0.48 & \\
\hline De Ridder ${ }^{23}$ & 60 & & 0.44 & & \\
\hline Sarica $^{22}$ & 10 & 1 & 0.07 & & \\
\hline \multicolumn{6}{|c|}{ Community setting } \\
\hline \multicolumn{6}{|c|}{ Uncoated } \\
\hline Cardenas $^{20}$ & 23 & & 0.14 & 0.14 & 0.47 \\
\hline \multicolumn{6}{|l|}{ Hydrophilic } \\
\hline Cardenas $^{20}$ & 22 & & 0.06 & 0.06 & \\
\hline \multicolumn{6}{|c|}{ Combined scenario } \\
\hline \multicolumn{6}{|l|}{ Uncoated } \\
\hline Cardenas $^{20}$ & 23 & & 0.14 & 0.40 & 0.92 \\
\hline Cardenas $^{21}$ & 114 & & 0.48 & & \\
\hline De Ridder 23 & 61 & & 0.38 & & \\
\hline Sarica $^{22}$ & 10 & 4 & 0.27 & & \\
\hline \multicolumn{6}{|l|}{ Hydrophilic } \\
\hline Cardenas $^{20}$ & 22 & & 0.06 & 0.37 & \\
\hline Cardenas $^{21}$ & 105 & & 0.48 & & \\
\hline De Ridder ${ }^{23}$ & 60 & & 0.34 & & \\
\hline Sarica $^{22}$ & 10 & 1 & 0.07 & & \\
\hline
\end{tabular}


Table 2 Haematuria rates (mean number of haematuria episodes per patient per year)

\begin{tabular}{|c|c|c|c|c|c|c|}
\hline Study & Patients & $\begin{array}{l}\text { Number of } \\
\text { events }\end{array}$ & Years & $\begin{array}{l}\text { Rate per patient } \\
\text { per year }\end{array}$ & Weighted mean & Rate ratio \\
\hline \multicolumn{7}{|l|}{ Uncoated } \\
\hline Cardenas $^{21}$ & 114 & 6 & 0.5 & 0.11 & 0.29 & 1.35 \\
\hline De Ridder ${ }^{23}$ & 59 & 32 & 1 & 0.54 & & \\
\hline Sarica $^{22}$ & 10 & 1 & 0.1151 & 0.87 & & \\
\hline \multicolumn{7}{|l|}{ Hydrophilic } \\
\hline Cardenas $^{21}$ & 105 & 14 & 0.5 & 0.27 & 0.39 & \\
\hline De Ridder ${ }^{23}$ & 55 & 38 & 1 & 0.69 & & \\
\hline Sarica $^{22}$ & 10 & 0 & 0.115 & 0.00 & & \\
\hline
\end{tabular}

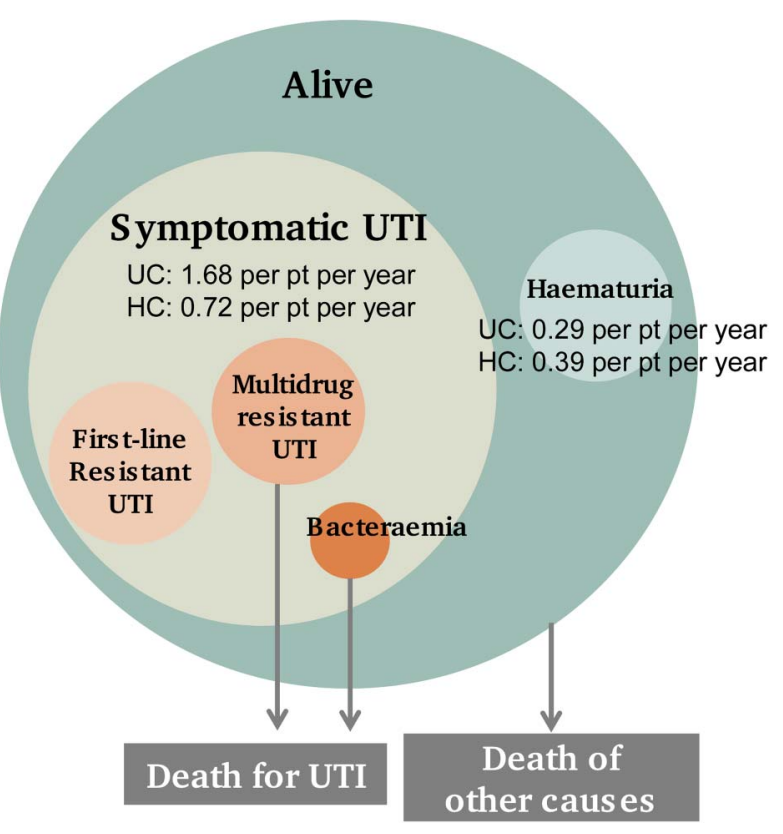

Figure 1 Simplified Markov model representation. Patients start the Markov process in the 'Alive' state, where they can remain or move to the 'Symptomatic UTI' or 'Haematuria' states. These are considered sub-states of the 'Alive' state since they last less than 1 year. The model accounts for the possibility of patients dying from causes other than UTI (death of other causes). HC, hydrophilic coated catheters; pt, patient; UC, uncoated catheters; UTI, urinary tract infection.

The model is mainly based on the structure presented by Bermingham $e t a l^{3}$ and focuses on short-term consequences of UTIs and haematuria. In contrast to Bermingham et $a l^{3}{ }^{3}$ who use the annual probability of experiencing at least one UTI, the current model incorporates the estimation of mean number of UTIs per patient and month as reported for Clark et al to give a more precise estimate of costs and patients' quality of life.

A 1-year Markov cycle length and a lifetime horizon were chosen for baseline analysis. To improve the accuracy of the results, a half-cycle correction was performed. The model was developed and analysed in Microsoft Excel.

\section{Model quantification}

As described above, monthly rates of 0.14 and 0.06 were estimated for symptomatic UTIs in patients using uncoated catheters and hydrophilic coated catheters in the community setting, respectively. These data translate into 1.68 and 0.72 events per year and patient, respectively. For haematuria, 0.29 and 0.39 episodes per year and patient were estimated for uncoated and hydrophilic coated catheters, respectively.

The probabilities of clinical failure after treatment for symptomatic UTI reported by Clark et at were mainly based on expert opinions, so annual transition probabilities as presented by Bermingham $e t a \hat{l}^{\hat{3}}$ were preferred. The annual probabilities of clinical failure, leading to first-line/multidrug-resistant UTI or bacteraemia, were applied to the mean number of symptomatic UTIs experienced by the patients over 1 year using uncoated or hydrophilic coated catheters.

As no further transition probabilities were found in literature, the model assumed that 'multidrug-resistant UTI' state also included healthcare resource consumption related to 'first-line-resistant UTI' state.

Standardised mortality ratios for men and women with SCI were retrieved by Lidal et $a l^{26}$ Mortality rates were further adjusted for age and gender according to Italian mortality tables (ISTAT).

A summary of transition probabilities and model parameters is presented in tables 3 and 4, respectively (for cost data and event durations, see the details in the Results section-Healthcare resource consumption and costs).

\section{Healthcare resource consumption and costs}

As the analysis was performed from the Italian Healthcare System perspective, all costs related to the consumption of direct healthcare resources were estimated and expressed in euro (2015 value).

Clinical pathways and healthcare resource consumption for the management of symptomatic UTIs, first-line-resistant UTIs, multidrug-resistant UTIs, haematuria episodes and bacteraemia were estimated by studyspecific questionnaire to urologists and neuro-urologists. All the clinicians $(\mathrm{N}=25)$ belonging to the NUS team (Italian spinal neuro-urologist group) of Fondazione 
Table 3 Transition probabilities matrix

\begin{tabular}{llll}
\hline Health state & Transition to & Annual transition probability & Reference \\
\hline Symptomatic UTI & First-line-resistant UTI & 0.083 & 3 \\
Symptomatic UTI & Multidrug-resistant UTI & 0.07 & 3 \\
Symptomatic UTI & Bacteraemia & 0.036 & 3 \\
Multidrug-resistant UTI & Death from UTI & 0.026 & 3 \\
Bacteraemia & Death from UTI & 0.077 & 3 \\
\hline UTI, urinary tract infection. & &
\end{tabular}

Table 4 Model parameters with related sources

\begin{tabular}{|c|c|c|}
\hline Parameter & Base-case value & Reference \\
\hline \multicolumn{3}{|l|}{ Population } \\
\hline Start age (years) & 40 & 25 \\
\hline Proportion men & $80 \%$ & 25 \\
\hline \multicolumn{3}{|l|}{ Utility coefficients for the health states/events } \\
\hline Alive & 0.831 & 3 \\
\hline Symptomatic UTI & 0.782 & 3 \\
\hline First-line-resistant UTI & 0.76 & 3 \\
\hline Multidrug-resistant UTI & 0.738 & 3 \\
\hline Bacteraemia & 0.716 & 3 \\
\hline Haematuria & 0.738 & $\begin{array}{l}\text { Assumed equal to multidrug- } \\
\text { resistant UTI }\end{array}$ \\
\hline \multicolumn{3}{|c|}{ Mean number of events per patient per year (uncoated catheters) } \\
\hline Symptomatic UTI & 1.68 & 20 \\
\hline Haematuria & 0.29 & $21-23$ \\
\hline \multicolumn{3}{|l|}{ Rate ratios } \\
\hline Symptomatic UTI (hydrophilic vs uncoated) & 0.47 & 20 \\
\hline Haematuria (hydrophilic vs uncoated) & 1.35 & $21-23$ \\
\hline Standardised mortality ratios for $\mathrm{SCI}$ patients & men 1.8, women 4.9 & 26 \\
\hline \multicolumn{3}{|l|}{ Costs } \\
\hline Unit cost, standard catheter & $€ 0.25$ & Tender data for Italy \\
\hline Unit cost, hydrophilic catheter & $€ 1.70$ & Tender data for Italy \\
\hline Alive (annual cost) & $€ 954.48$ & E-survey and official tariffs \\
\hline Symptomatic UTI & $€ 1091.86$ & E-survey and official tariffs \\
\hline First-line-resistant UTI & $€ 401.20$ & E-survey and official tariffs \\
\hline Multidrug-resistant UTI & $€ 775.36$ & E-survey and official tariffs \\
\hline Bacteraemia & $€ 3664.16$ & E-survey and official tariffs \\
\hline Haematuria & $€ 106.10$ & E-survey and official tariffs \\
\hline Death for bacteraemia & $€ 6057.70$ & E-survey and official tariffs \\
\hline Death for multidrug-resistant UTI & $€ 9721.86$ & E-survey and official tariffs \\
\hline \multicolumn{3}{|l|}{ Events duration (days) } \\
\hline Symptomatic UTI & 4 & E-survey \\
\hline First-line-resistant UTI & 8 & E-survey \\
\hline Multidrug-resistant UTI & 16 & E-survey \\
\hline Bacteraemia & 37 & DRG 576 \\
\hline Haematuria & 2 & E-survey \\
\hline Bacteraemia, if leading to death & 65 & DRG 575 \\
\hline Pre-death multidrug-resistant UTI hospitalisation & 65 & DRG 575 \\
\hline
\end{tabular}

Italiana Continenza (Italian Continence Foundation), ${ }^{27}$ which treat the highest volumes of patients across Italy, received access to a web version of the questionnaire (developed with Qualtrics software) between 15 July 2015 and 15 October 2015 (a printed version of the questionnaire is available on request). The questionnaire included four sections: (1) introduction with a case vignette, ${ }^{28}$ (2) patient monitoring (relevant annual examinations, laboratory tests, visits, inpatient stays and drugsirrespective of catheter type), (3) management of UTIs, bacteraemia and haematuria, and (4) future scenarios of catheter use. On the basis of their clinical experience, clinicians were asked to estimate healthcare usage, for example, the percentage of patients involved, regimen applied (outpatient, day-hospital or inpatient stay), daily dose and duration of drugs for general management 
and/or for management of an episode of UTI, bacteraemia and haematuria (drug costs are generally provided by an administrative office within the hospital).

The last section of the questionnaire included a forecast of possible future scenarios (1, 3 and 5 years) of usage of uncoated and hydrophilic coated catheters in Italy.

The results from the questionnaires were summarised to estimate healthcare resource usage. For each healthcare resource (examination, visit, hospitalisation, etc.) reported, a weighted mean was calculated on the basis of the number of responders.

The cost of resource consumption for the different events was calculated by multiplying the quantity of resources consumed by unit costs derived from official sources, that is, diagnosis-related groups' (DRGs) reimbursement for hospitalisations, official tariffs for outpatient services, and hospital prices for drugs. When hospital prices for drugs were missing, a search was performed through the Italian Pharmaceutical Database (http://www.federfarma.it) reporting cost data for the National Healthcare Service.

Four catheters per day and patient were assumed, as this was the reimbursement level provided by the local health agencies. The unit cost was estimated from tender data at $€ 1.70$ and $€ 0.25$ for hydrophilic coated and uncoated catheters, respectively. In Italy, the lubricant gel for uncoated catheters is paid for by the patients, so this cost was omitted in the model.

During hospital stays, catheter costs are assumed included in the DRG reimbursement, excluding the need for additional device costs in the model.

\section{Quality-of-life estimates}

The search for utility coefficients for SCI patients performing IC was performed through PubMed, Embase, Web of Science databases and the Cost-Effectiveness Analysis Registry. ${ }^{29}$ Two studies ${ }^{30}{ }^{31}$ and a review ${ }^{32}$ were found that reported utility values for SCI patients experiencing UTIs. The first one ${ }^{30}$ reported utility values (estimated by HUI-Mark III health status classification system) of 0.28 and 0.15 for no/mild UTI and moderate/significant UTI, respectively. The second study ${ }^{31}$ reported utility values for UTI of 0.58 and 0.60 estimated by SF36 and SF12 questionnaires, respectively. The review ${ }^{32}$ included an additional study conducted by Vogel and Zebracki from which utility values of 0.831 , 0.782 and 0.738 were estimated for no UTI, UTI and severe UTI, respectively. From the database search, no utility values were found for haematuria and bacteraemia health states.

Additional utility values were retrieved from Bermingham $e t a \hat{l}^{\hat{3}}$ and Clark $e t a l^{4}$ All values are summarised in online supplementary table S1.

The model included utility values referred to in the study by Bermingham $e t a \hat{l}$ (see table 4).

For haematuria, a utility value of 0.738 (as for multidrug-resistant UTI state) was assumed.
The duration of the different events was estimated from the pharmacological treatment duration reported by the questionnaires, with the exception of both multidrug-resistant UTI and bacteraemia leading to death for which the length-of-stay threshold of the related DRGs was considered.

\section{Analyses}

Both incremental cost-effectiveness and cost-utility ratios (ICER and ICUR) of hydrophilic coated versus uncoated catheters were calculated by dividing the incremental cost by the incremental health improvement. Life years, QALYs and costs were discounted with a $3.5 \%$ yearly rate. $^{33}$ Transition probabilities, costs and utilities were entered into the model along with a distribution: beta for utilities and proportions of patients experiencing different kinds of UTIs, log-normal for relative risks and gamma for costs. Deterministic and probabilistic sensitivity analyses (PSA) were performed to test the robustness of the model. Univariate analyses were performed according to the main parameters; second-order Monte-Carlo analysis (1000 simulations) was conducted and the related acceptability curve was plotted.

Further analyses were performed that considered UTI rates for (1) hospital period and (2) combined (hospital plus community) scenario (based on data presented in table 1). Since UTI rates per patient per month vary across the retrieved studies, different scenario analyses were performed that considered data input from each study separately to evaluate heterogeneity. The same was performed for episodes of haematuria (based on data presented in table 2).

\section{Budget Impact Analysis}

Based on the conclusion from the CEA model, a companion budget impact model ${ }^{34}$ was developed to address hypothetical changes to the Italian Healthcare Service budget considering an increased usage of hydrophilic coated catheters.

In order to perform the BIA, a review of epidemiological data focused on SCI patients performing IC was carried out.

The prevalence of SCI patients in Italy resulted in the range $60000-70000$ according to a national registry, ${ }^{35}$ while the incidence (data from the Italian registry) showed a decrease from 20-25 to 7.8 per million inhabitants. Based on the study by Zlatev $e t a l^{36}$ it was assumed that $60 \%$ of patients perform IC. The total number of prevalent patients with SCI performing IC in Italy was estimated to be about $39000(65000 \times 60 \%)$, while the total number of incident patients was about 285. It was assumed that the distribution of the incident population was the same as that of the prevalent population (mean age of 40 years, $80 \%$ men).

The current scenario of patient distribution between the two devices under consideration was estimated from clinical input as $20 \%$ uncoated and $80 \%$ hydrophilic coated catheters. The estimation of the new scenario, 
including an increased proportion of hydrophilic coated catheters in the years, was based on key opinion leaders' replies to the questionnaire.

The cost of the current and new scenarios was determined by multiplying the cost for each intervention by the proportion of the eligible population using it, taking into account both prevalent and subsequent yearly incident cohorts. Financial streams were presented as undiscounted costs, since the focus of the analysis was expected budget at each point. ${ }^{34}$

\section{RESULTS}

\section{Healthcare resource consumption and costs}

A total of 9 of 25 clinicians completed the questionnaire, representing institutions with the highest volumes of treated SCI patients in Italy. The estimated healthcare resource usage is reported in online supplementary table S2. Reported care pathways were consistent with previous published literature. ${ }^{37}$

The 'Alive' health state in the model refers to usual patient year including control visits, examinations or hospitalisations for causes other than UTIs (eg, urethral strictures, bladder stones). All other health states consider healthcare resources consumption for management of a single event (eg, symptomatic UTI, haematuria, bacteraemia). For drugs, the mean dosage per patient was reported together with the proportion of administered patients.

Unit costs related to the healthcare resource consumption are summarised in online supplementary table S3.

The estimated event durations were 2 days for haematuria, 4 days for symptomatic UTI, additional 8 days for first-line-resistant UTI, additional 8 days for multidrugresistant UTI (total $4+8+8=20$ days), 37 days for hospitalisation for bacteraemia (DRG 576) and 65 days for infection leading to death (DRG 575).

In case of bacteraemia leading to patient death, the healthcare resources related to 'infection leading to patient death' (see online supplementary table S2) were applied (the management of the episode of bacteraemia is included in the DRG 575).

Summaries of event durations and costs estimated for the different health states/events are included in table 4.

\section{Baseline results}

Deterministic and probabilistic results were obtained from the model. It estimated an average life expectancy of 18.3 years (15.2 QALYs) for a study population using hydrophilic coated catheters and 17.3 years (14.3 QALYs) for a study population using uncoated catheters. The mean lifetime costs per patient were $€ 82915$ and $€ 62457$ for hydrophilic coated and uncoated catheters, respectively. For hydrophilic coated catheters, this resulted in an ICER of $€ 20761$ and an ICUR of $€ 24405$ (table 5-deterministic results). Although there is no official cost-effectiveness threshold for Italy, the reported proposed thresholds vary between $€ 25000-€ 40000,{ }^{38}$ $€ 36500,{ }^{39} € 60000,{ }^{40}$ and $€ 66400$ (three times the Italian gross domestic product per capita according to the WHO) ${ }^{41}{ }^{42}$ This suggests that the ICER/ICUR for hydrophilic coated catheters is lower than recommended threshold values and thus could be considered a cost-effective option.

Considering a lifetime horizon, hydrophilic coated catheters may reduce the frequency of UTIs by about $50 \%$ (from 48 to 24 ) in comparison to uncoated catheters. Considering the significant impact of UTIs, which account for about $23 \%$ to $63 \%$ of the total lifetime cost for SCI patients practicing intermittent catheterisation, prevention is of high importance.

A PSA was performed to account for uncertainty in cost-effectiveness calculations (online supplementary table S4 summarises the main model parameters with related probability distributions). Probabilistic model results are included in table 5 .

The acceptability curve obtained from the Monte-Carlo simulation is shown in figure 2 for the ICUR. Given the varying Italian threshold values of $€ 25$ 000-40 000, €36 500, €60 000 and $€ 66400$, hydrophilic coated catheters have about a $47-86 \%, 77 \%, 97 \%$ and $98 \%$ probability of being cost-effective, respectively. Considering the UK-specific threshold value of $£ 20000$ $£ 30000$ recommended by $\mathrm{NICE}^{33}$ (equal to €26 400$€ 39600$ at an exchange rate of 1.32), hydrophilic coated catheters have a $48-86 \%$ probability of being costeffective.

The scenario analyses performed considering weighted UTI rates for hospital period and combined (hospital plus community) settings resulted in ICURs equal to $€ 11908 /$ QALY (ICER $€ 10097 / \mathrm{LY}$ ) and $€ 97$ 019/QALY (ICER €82 188/LY), respectively.

The additional scenario analyses conducted considering UTI rates from single studies, as reported in table 1, showed ICUR values for the hospital period that were equal to $€ 11240 /$ QALY and $€ 17368 /$ QALY, based on

\begin{tabular}{llllllllll} 
Table 5 & \multicolumn{1}{l}{ Summary of the model results } \\
\hline Results & Catheter & Cost $(€)$ & $\Delta$ Cost $(€)$ & LY & $\Delta$ LY & QALYs & $\Delta$ QALYs & ICER ( $€ / L Y)$ & ICUR ( $€ /$ QALY) \\
\hline Deterministic & Uncoated & 62457 & & 17.299 & & 14.332 & & & \\
& Hydrophilic & 82915 & 20459 & 18.284 & 0.985 & 15.170 & 0.838 & 20761 & 24405 \\
Probabilistic & Uncoated & 62357 & & 17.300 & & 14.329 & & & \\
& Hydrophilic & 82971 & 20614 & 18.276 & 0.977 & 15.158 & 0.830 & 21110 & 24840 \\
\hline ICER, incremental cost-effectiveness ratio; ICUR, incremental cost-utility ratio; LY, life year; QALYs, quality-adjusted life years.
\end{tabular}




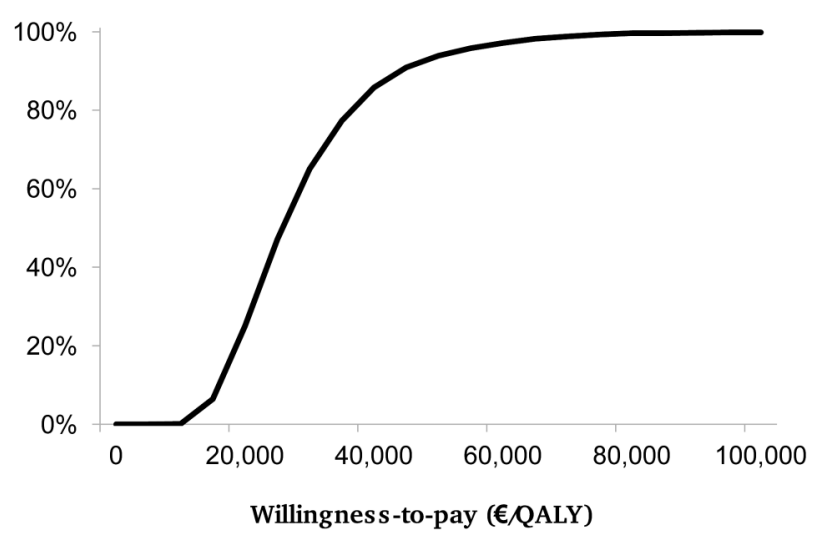

Figure 2 ICUR acceptability curve. ICUR, incremental cost-utility ratio; QALY, quality-adjusted life year.

data from Cardenas et al $(2011)^{21}$ and De Ridder et al $(2005),{ }^{23}$ respectively. Concerning the combined scenario, ICURs obtained were €21 184/QALY and €68 979/ QALY based on data from Cardenas and Hoffman $(2009)^{20}$ and De Ridder et al (2005), ${ }^{23}$ respectively. Only data from Cardenas et al (2011) ${ }^{21}$ showed the dominance of uncoated catheters, while data from Sarica et al $(2010)^{22}$ showed hydrophilic catheter dominance for both hospital and hospital-plus-community settings.

Scenario analyses considering haematuria rates from single studies, as reported in table 2, showed limited variations in the ICUR, which ranged from $€ 22$ 000/QALY (data from Sarica et al $(2010)^{22}$ ) to $€ 24569 /$ QALY (data from De Ridder et al $\left.(2005)^{23}\right)$, respectively.

One-way sensitivity analyses were performed for the ICUR on the main model parameters. The results for the 10 parameters responsible for the main ICUR variations are presented in a tornado diagram in figure 3 (see online supplementary table S5 for complete results). The parameters with the greatest impact on ICUR were the relative risk (rate ratio) of developing a symptomatic UTI (for hydrophilic catheters vs uncoated catheters), the mean number of symptomatic UTIs per patient and year for uncoated catheters, the unit cost for hydrophilic catheters and the number of catheters used per day. For example, a rate ratio higher than 0.70 for developing a symptomatic UTI would result in ICUR values over $€ 60000$. Hydrophilic coated catheters are the dominant choice if the unit cost is $€ 0.85$ or lower, but if the unit cost is $€ 2.55$, the ICUR exceeds $€ 50000$. Also, lowering the utility value for the 'Alive' health state to 0.42 results in an ICUR above $€ 65000$.

\section{Budget impact analysis}

As hydrophilic coated catheters are likely to be a costeffective strategy, a BIA was performed to consider a new scenario with an increasing proportion of users of these advanced devices among patients performing IC in the next years. The proportions for possible future usages were estimated by the questionnaires. Focusing on uncoated and hydrophilic coated catheters only, the clinicians reported possible proportions of hydrophilic coated catheter use of $83 \%, 88 \%$ and $89 \%$ after 1,3 and 5 years, respectively.

Table 6 reports the mean yearly cost per patient for both uncoated and hydrophilic coated catheters as calculated from the CEA model. Costs are presented for the following four sub-categories: patient monitoring (ie, control visits/examinations, etc.), management of UTIs, management of haematuria episodes, and catheters. The highest costs for uncoated catheters are related to the management of UTIs, while the highest costs for hydrophilic coated catheters are reported for the catheters themselves.

Table 7 reports the annual cost for SCI patients performing IC with either uncoated or hydrophilic coated catheters with related number of users (year 0: prevalent cohort, following years: incident cohorts), for both current and new scenarios. For both catheter types, the total cost per year has been weighted according to the proportion of use (ie, $80 \%$ hydrophilic coated and $20 \%$ uncoated catheters for all years in the current scenario

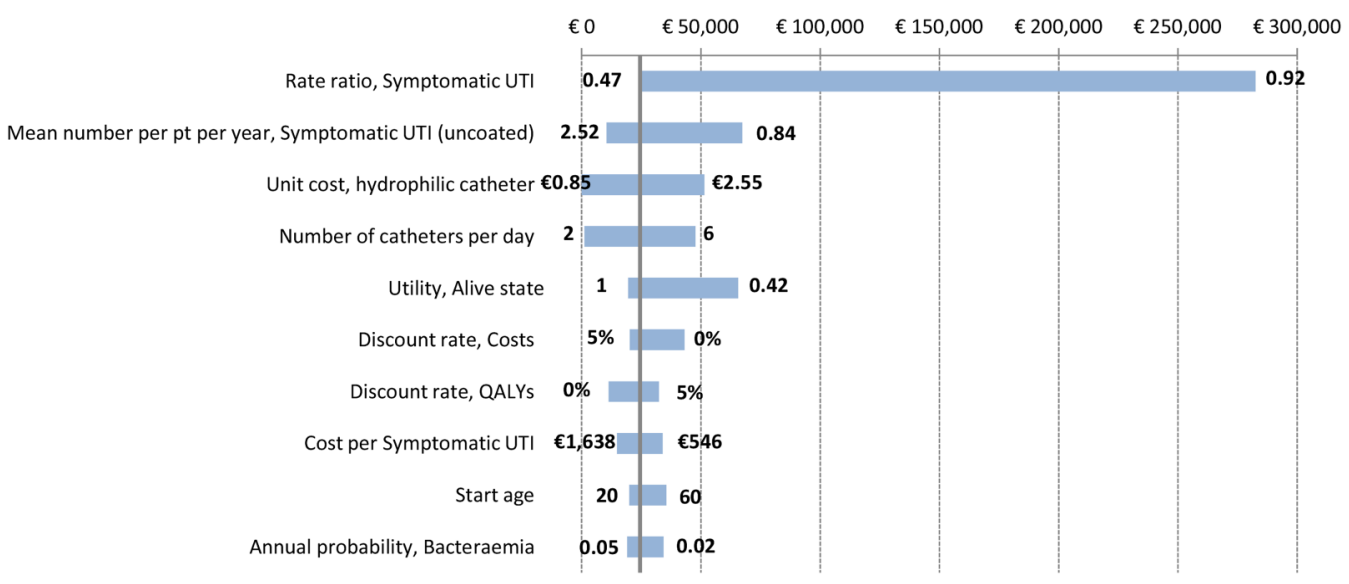

Figure 3 Tornado diagram showing one-way sensitivity analyses on ICUR value (€24 405). Upper and lower limits of variables' values referring to the ICUR extremes are indicated next to the bars. ICUR, incremental cost-utility ratio; pt, patient; QALYs, quality-adjusted life years; UTI, urinary tract infection. 
Table 6 Detailed mean costs per patient for uncoated and hydrophilic coated catheters for the first 5 years

\begin{tabular}{|c|c|c|c|c|c|c|c|c|c|c|}
\hline \multirow[b]{2}{*}{ Year } & \multicolumn{5}{|c|}{ Uncoated catheters (UC) } & \multicolumn{5}{|c|}{ Hydrophilic coated catheters (HC) } \\
\hline & $\begin{array}{l}\text { UC patient } \\
\text { monitoring }\end{array}$ & $\begin{array}{l}\text { UC } \\
\text { UTIs }\end{array}$ & $\begin{array}{l}\text { UC } \\
\text { haematuria }\end{array}$ & $\begin{array}{l}\text { UC } \\
\text { catheters }\end{array}$ & $\begin{array}{l}\text { UC total } \\
\text { cost }\end{array}$ & $\begin{array}{l}\text { HC patient } \\
\text { monitoring }\end{array}$ & $\begin{array}{l}\text { HC } \\
\text { UTIs }\end{array}$ & $\begin{array}{l}\text { HC } \\
\text { haematuria }\end{array}$ & $\begin{array}{l}\text { HC } \\
\text { catheters }\end{array}$ & $\begin{array}{l}\text { HC total } \\
\text { cost }\end{array}$ \\
\hline 0 & $€ 950$ & $€ 2250$ & $€ 31$ & $€ 361$ & $€ 3591$ & $€ 951$ & $€ 1060$ & $€ 41$ & $€ 2468$ & $€ 4520$ \\
\hline 1 & $€ 940$ & $€ 2227$ & $€ 30$ & $€ 357$ & $€ 3554$ & $€ 945$ & $€ 1053$ & $€ 41$ & $€ 2452$ & $€ 4491$ \\
\hline 2 & $€ 929$ & $€ 2203$ & $€ 30$ & $€ 353$ & $€ 3515$ & $€ 939$ & $€ 1046$ & $€ 41$ & $€ 2436$ & $€ 4461$ \\
\hline 3 & $€ 919$ & $€ 2179$ & $€ 30$ & $€ 349$ & $€ 3477$ & $€ 933$ & $€ 1039$ & $€ 41$ & $€ 2419$ & $€ 4431$ \\
\hline 4 & $€ 909$ & $€ 2154$ & $€ 29$ & $€ 345$ & $€ 3438$ & $€ 926$ & $€ 1031$ & $€ 40$ & $€ 2401$ & $€ 4399$ \\
\hline 5 & $€ 898$ & $€ 2129$ & $€ 29$ & $€ 341$ & $€ 3398$ & $€ 919$ & $€ 1024$ & $€ 40$ & $€ 2383$ & $€ 4366$ \\
\hline
\end{tabular}

and increasing percentage of use of hydrophilic catheters in the years in the new scenario). The last two columns summarise the total national healthcare budget and the yearly incremental cost. An increasing use of hydrophilic coated catheters results in an increase of the total budget of about $€ 12$ million in the next 5 years.

\section{DISCUSSION}

IC is considered the method of choice for the management of neurogenic bladder dysfunctions. Patients performing IC entail a substantial economic burden on the healthcare system, as infections and urethral trauma are common and result in frequent hospitalisations and high morbidity. Although different catheters are available with various characteristics in terms of medical safety, treatment functionality, patient comfort and environmental performance, there is currently no robust consensus as to which catheter type is the best. Recent meta-analyses investigating the impact of different catheters types on UTI rate and haematuria reported conflicting results. One study ${ }^{43}$ concluded that hydrophilic coated catheters are associated with a significant reduction in the risk of UTI and haematuria compared to non-hydrophilic catheters while another study was unable to differentiate between catheter types and techniques. $^{2}$

The aim of this study was to conduct cost-effectiveness and budget impact analyses of different catheters used for IC. The results were meant to support the process for deciding how to allocate scarce healthcare resources and maximise patients' health while controlling costs. In Italy, the provision of disposable medical devices for daily repeated use, such as catheters for IC, is currently regulated by the Ministry of Health $(\mathrm{MoH}),{ }^{44}$ which defines a list of medical devices supplied directly to patients and reimbursed by the Italian NHS. In recent times, the coverage of medical devices has been the subject of debates in Italy. The $\mathrm{MoH}$ has decided that more information is needed on the value contribution of medical devices both to patients and to the healthcare systems. For this reason, a National Health Technology Assessment Programme has been developed that refers to CEA as the main decision tool in measuring the incremental value of innovative technologies in comparison to the standard of care. ${ }^{45-47}$

Considering a lifetime perspective, hydrophilic coated catheters resulted in an ICUR of €24 405/QALY and an ICER of $€ 20761 / \mathrm{LY}$. Accordingly, hydrophilic coated catheters were likely to be considered cost-effective in comparison to uncoated catheters, given the available range of thresholds values proposed for Italy (from $€ 25000$ to $€ 66400)$. PSA supported this findings: considering the Italian threshold values of $€ 25000-€ 40000$, $€ 36500$, $€ 60000$ and $€ 66$ 400, hydrophilic coated catheters showed about a $47-86 \%, 77 \%, 97 \%$ and $98 \%$ probability of being cost-effective, respectively.

The base-case findings are in line with conclusions reported by Clark and colleagues, ${ }^{4}$ who considered a UK setting and a cost-effectiveness threshold of $£ 30000$ (about $€ 40000$ ). However, the results differ from the report by Bermingham et $a l^{3}{ }^{3}$ who concluded that uncoated catheters are the most cost-effective when compared to all the other catheter types. This discrepancy is likely related to the difference in selecting studies and data for the underlying meta-analysis investigating UTI risk. Bermingham et al used data from a meta-analysis that estimated the risk of experiencing at least one UTI for each catheter type. Since there could be a great variation in the number of UTIs experienced by each patient, this assumption could potentially have hidden a risk-reducing efficacy related to hydrophilic coated catheters. A study ${ }^{48}$ evaluating self-reported catheter practices and associated problems for people mainly performing IC with uncoated catheters found an annual rate of 2.3 (95\% CI 1.8 to 3) symptomatic UTIs treated with an antibiotic.

When a lower cost-effectiveness threshold was considered (ie, $£ 20000$ to about $€ 26400$ ), the probability that hydrophilic coated catheters may be a cost-effective choice was about $50 \%$, partially supporting the conclusions presented by Bermingham $e t \mathrm{al}^{3}$

Different from Clark et $a l,{ }^{4}$ the present CE model focused only on short-term consequences of symptomatic UTIs, excluding lifetime effects on renal function. Since the probability of developing UTIs was found to be lower for hydrophilic coated catheters versus uncoated ones, this suggests that results are conservative 


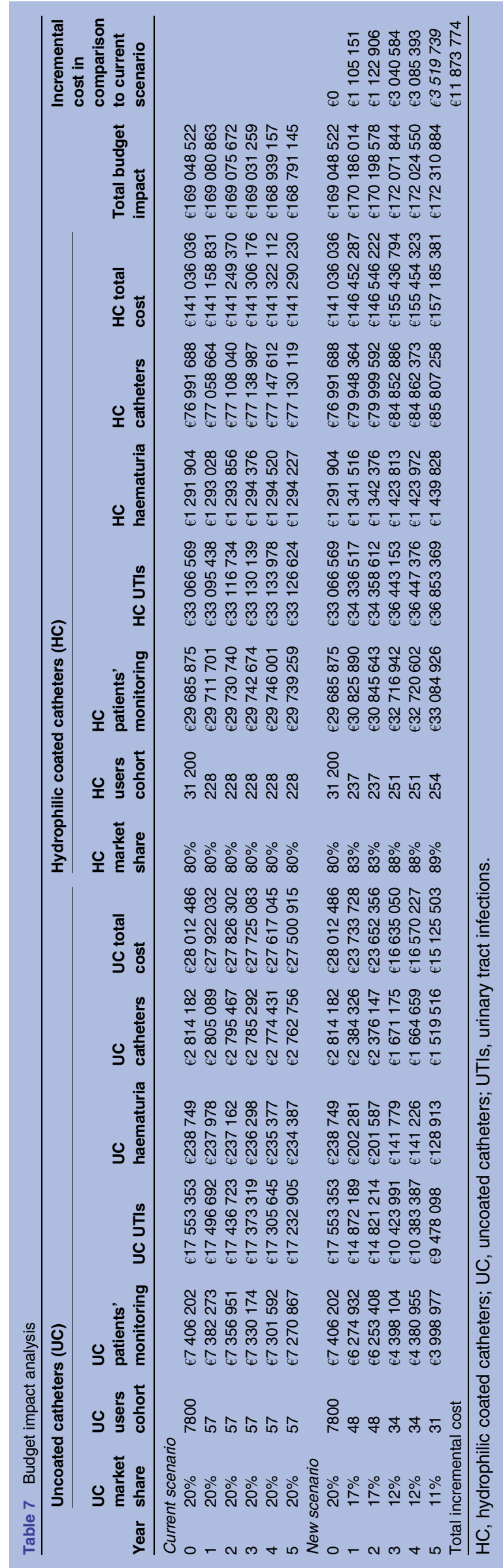

estimates of the CE results. As a consequence, the scenario analyses considering community setting and hospital-plus-community settings together reported higher ICERs and ICURs in comparison to the findings of the study cited above.

Another difference is related to the cost of the two devices. While in UK the cost of an uncoated catheter is slightly lower than the cost of a hydrophilic coated one, in Italy the cost of uncoated catheters is very low, about $25 \%$ of the cost of the hydrophilic coated catheter. The increased cost of hydrophilic coated catheters is partially offset by the cost savings due to the lower number of UTIs that develop.

Our study is a CEA comparing hydrophilic coated to uncoated catheters that also includes a BIA. The BIA is considered to add important information for decisionmakers who need to estimate the impact on healthcare expenditures of introducing new health technologies in regular practice.

This study estimated the consumption of healthcare resources by soliciting expert opinions with the aim of providing real-world costing data. This is especially important for medical devices, since their use in regular practice often differs from that established in experimental settings. ${ }^{49}$ Also, the fact that the consumption of healthcare resources has been represented in natural units—as suggested by the EUnetHTA guidelines ${ }^{50}$ — will allow cost adjustment to other countries.

This study has some limitations. First of all, clinical effectiveness data were derived from few RCTs with fewer than 50 participants and with variations in length of follow-up and definitions of UTI. Moreover, the rates of events per patient per month varied across the studies and the calculated weighted means may not be fully representative of the Italian scenario.

The model focused mainly on complications such as UTI and haematuria, for which different rates were estimated for hydrophilic coated and uncoated catheters. UTIs are recognised as the most frequent complications, while epididymitis and urethritis are relatively rare.$^{51}$ To the best of our knowledge, there are no randomised controlled data on other complications for different catheter types. However, observational studies reported fewer traumas and urethral inflammations for hydrophilic coated catheters that would potentially increase their cost-effectiveness on a lifetime perspective. ${ }^{52} 53$

As regards the estimation of the healthcare resources, it must be noted that data derived from self-reported questionnaires may be limited by varying recollection and poor generalisability. Variables derived from prospective observational multi-centre studies would increase the validity of the current model. Observational studies would also serve to confirm clinical evidence of the comparative effectiveness of catheters in addition to RCTs.

Overall, the analysis is based on varying levels of evidence and assumptions, and the results need to be considered cautiously. 
The findings of this study support the use of hydrophilic coated catheters but are limited to costs from a healthcare perspective. A broader evaluation, also including costs from a societal perspective, would increase the understanding of the economic sustainability of these devices.

Acknowledgements The authors would like to thank Dr Kristian Neovius for his support in the cost-effectiveness model design, Dr Michele Spinelli who made valuable suggestions in the questionnaire development and the clinicians who participated in the data collection: Elena Andretta, Sauro Biscotto, Mirella Cianfrocca, Gabriella Fizzotti, Alberto Manassero, Daniele Minardi, Oreste Risi, Sandro Sandri and Francesco Savoca.

Collaborators Gabriella Fizzotti provided clinical advice for the manuscript preparation; Michele Spinelli provided suggestions in the questionnaire development.

Contributors CR is responsible for study concept and design, analysis and interpretation of data and drafting of the manuscript. RT is responsible for study supervision, acquisition of funding and critical revision of the manuscript.

Funding This study was funded by ASBM Srl through an unrestricted grant to CERGAS, Bocconi University, Via Roentgen 1, 20136 Milan, Italy. The study sponsor had no role in the study design; the collection, analysis and interpretation of data; the writing of the report; or the decision to submit the article for publication. No interferences occurred in carrying out the research project, or in writing the manuscript that is the sole responsibility of the authors.

Competing interests All authors have completed the Unified Competing Interests form at www.icmje.org/coi_disclosure.pdf (available on request from the corresponding author) and declare that CeRGAS Bocconi has support from ASBM Srl for the submitted work.

Provenance and peer review Not commissioned; externally peer reviewed.

Data sharing statement Additional data are available in online supplementary appendix.

Open Access This is an Open Access article distributed in accordance with the Creative Commons Attribution Non Commercial (CC BY-NC 4.0) license, which permits others to distribute, remix, adapt, build upon this work noncommercially, and license their derivative works on different terms, provided the original work is properly cited and the use is non-commercial. See: http:// creativecommons.org/licenses/by-nc/4.0/

\section{REFERENCES}

1. Cardarelli WJ. Managed care aspects of managing neurogenic bladder/neurogenic detrusor overactivity. Am J Manag Care 2013;19 (Suppl):s205-8.

2. Prieto J, Murphy CL, Moore KN, et al. Intermittent catheterisation for long-term bladder management. Cochrane Database Syst Rev 2014; (9):CD006008.

3. Bermingham SL, Hodgkinson S, Wright S, et al. Intermittent self catheterisation with hydrophilic, gel reservoir, and non-coated catheters: a systematic review and cost effectiveness analysis. $B M J$ 2013;346:e8639.

4. Clark JF, Mealing SJ, Scott DA, et al. A cost-effectiveness analysis of long-term intermittent catheterisation with hydrophilic and uncoated catheters. Spinal Cord 2016;54:73-7.

5. Bakke A, Digranes A, Høisaeter PA. Physical predictors of infection in patients treated with clean intermittent catheterization: a prospective 7-year study. Br J Urol 1997;79:85-90.

6. Turi $\mathrm{MH}$, Hanif $\mathrm{S}$, Fasih Q, et al. Proportion of complications in patients practicing clean intermittent self-catheterization (CISC) vs indwelling catheter. J Pak Med Assoc 2006;56:401-4.

7. Nicolle LE. Urinary tract infections in patients with spinal injuries. Curr Infect Dis Rep 2014;16:390.

8. Ciani O, Grassi D, Tarricone R. An economic perspective on urinary tract infection: the "costs of resignation". Clin Drug Investig 2013;33:255-61.
9. Lai KK, Fontecchio SA. Use of silver-hydrogel urinary catheters on the incidence of catheter-associated urinary tract infections in hospitalized patients. Am J Infect Control 2002;30:221-5.

10. Karchmer TB, Giannetta ET, Muto CA, et al. A randomized crossover study of silver-coated urinary catheters in hospitalized patients. Arch Intern Med 2000;160:3294-8.

11. McNutt R, Johnson TJ, Odwazny R, et al. Change in MS-DRG assignment and hospital reimbursement as a result of Centers for Medicare \& Medicaid changes in payment for hospital-acquired conditions: is it coding or quality? Qual Manag Health Care 2010;19:17-24.

12. Saint S. Clinical and economic consequences of nosocomial catheter-related bacteriuria. Am J Infect Control 2000;28:68-75

13. Tambyah PA, Knasinski V, Maki DG. The direct costs of nosocomial catheter-associated urinary tract infection in the era of managed care. Infect Control Hosp Epidemiol 2002;23:27-31.

14. Anderson DJ, Kirkland KB, Kaye KS, et al. Underresourced hospital infection control and prevention programs: penny wise, pound foolish? Infect Control Hosp Epidemiol 2007;28:767-73.

15. Bardsley A. Intermittent self-catheterisation in women: reducing the risk of UTIs. Br J Nurs 2014:23(Suppl 18):S20-9.

16. Heard L, Buhrer R. How do we prevent UTI in people who perform intermittent catheterization? Rehabil Nurs 2005;30:44-5, 61.

17. Bennett E. Intermittent self-catheterisation and the female patient. Nurs Stand 2002;17:37-42.

18. Igawa $Y$, Wyndaele JJ, Nishizawa O. Catheterization: possible complications and their prevention and treatment. Int $J$ Urol 2008;15:481-5.

19. http://www.sanita24.ilsole24ore.com/pdf2010/Sanita2/ Oggetti Correlati/Documenti/Dal-Governo/Allegato\%202\%20Ausili\% 20Monouso\%202015 Filigrana.pdf (accessed 10 Jul 2016)

20. Cardenas DD, Hoffman JM. Hydrophilic catheters versus noncoated catheters for reducing the incidence of urinary tract infections: a randomized controlled trial. Arch Phys Med Rehabil 2009;90: 1668-71.

21. Cardenas DD, Moore KN, Dannels-McClure A, et al. Intermittent catheterization with a hydrophilic-coated. catheter delays urinary tract infections in acute spinal cord injury: a prospective, randomized, multicenter trial. PM R 2011;3:408-17.

22. Sarica $\mathrm{S}, \mathrm{Akkoc} \mathrm{Y}$, Karapolat $\mathrm{H}$, et al. Comparison of the use of conventional, hydrophilic and gel-lubricated catheters with regard to urethral micro trauma, urinary system infection, and patient satisfaction in patients with spinal cord injury: a randomized controlled study. Eur J Phys Rehabil Med 2010;46:473-9.

23. De Ridder DJ, Everaert K, Fernández LG, et al. Intermitten catheterisation with hydrophilic-coated catheters (SpeediCath) reduces the risk of clinical urinary tract infection in spinal cord injured patients: a prospective randomised parallel comparative trial. Eur Urol 2005;48:991-5.

24. Vahr S, Cobussen-Boekhorst $\mathrm{H}$, Eikenboom J, et al. Catheterisation. Urethral intermittent in adults: dilatation, urethral intermittent in adults. Arnhem, The Netherlands: European Association of Urology Nurses (EAUN), 2013:96.

25. Euro Mediterranean Rehabilitation Summer School. http://www. emrss.it/ (accessed 10 Mar 2016).

26. Lidal IB, Snekkevik H, Aamodt G, et al. Mortality after spinal cord injury in Norway. $J$ Rehabil Med 2007;39:145-51.

27. http://www.contenuti-web.com/continenza/fondazione-0000409.html (accessed $10 \mathrm{Jul}$ 2016)

28. Fattore G, Torbica A. Cost and reimbursement of cataract surgery in Europe: a cross-country comparison. Health Econ 2008;17(1 Suppl): S71-82.

29. CEA Registry. https://research.tufts-nemc.org/cear4/ SearchingtheCEARegistry (accessed 10 Jul 2016).

30. Craven C, Hitzig SL, Mittmann N. Impact of impairment and secondary health conditions on health preference among Canadians with chronic spinal cord injury. J Spinal Cord Med 2012;35:361-70.

31. Lee BB, King MT, Simpson JM, et al. Validity, responsiveness, and minimal important difference for the SF-6D health utility scale in a spinal cord injured population. Value Health 2008;11:680-8.

32. Bermingham SL, Ashe JF. Systematic review of the impact of urinary tract infections on health-related quality of life. BJU Int 2012;110(Pt C):E830-6.

33. National Institute for Clinical Excellence. Guide to the Methods of Technology Appraisal. London: National Institute for Clinica Excellence, 2004.

34. Sullivan SD, Mauskopf JA, Augustovski F, et al. Budget impact analysis-principles of good practice: report of the ISPOR 2012 Budget Impact Analysis Good Practice II Task Force. Value Health 2014;17:5-14. 
35. GISEM-Italian Group for the Epidemiological Study of Spinal Cord Injuries. http://www.istud.it/superabile/lesione.asp (accessed $10 \mathrm{Jul}$ 2016).

36. Zlatev DV, Shem K, Elliott CS. How many spinal cord injury patients can catheterize their own bladder? The epidemiology of upper extremity function as it affects bladder management. Spinal Cord 2016;54:287-91.

37. Biardeau X, Corcos J. Intermittent catheterization in neurologic patients: update on genitourinary tract infection and urethral trauma. Ann Phys Rehabil Med 2016;59:125-9.

38. Associazione Italiana di Economica Sanitaria (AIES). Proposta di linee guida per la valutazione economica degli interventi sanitari. PharmacoEcon Ital Res Artic 2009;11:83-93.

39. Lucioni $\mathrm{C}$, Ravasio R. How to evaluate the results of a pharmacoeconomic study? PharmacoEcon Ital Res Artic 2004;6:121-30

40. Messori A, Santarlasci B, Trippoli S, et al. Clinical benefit and economic value: methodology and an economic application. PharmacoEcon Ital Res Artic 2003;5:53-67.

41. World Health Organization. World Health Organization: choosing Interventions that are Cost Effective (WHO-CHOICE). Geneva: World Health Organization, 2012.

42. http://www.who.int/choice/costs/CER levels/en (accessed $10 \mathrm{Ju}$ 2016).

43. Li L, Ye W, Ruan H, et al. Impact of hydrophilic catheters on urinary tract infections in people with spinal cord injury: systematic review and meta-analysis of randomized controlled trials. Arch Phys Med Rehabil 2013;94:782-7.

44. Ministerial Decree 27th August 1999, n.332, http://www gazzettaufficiale.it/eli/id/1999/09/27/099G0404/sg (accessed $10 \mathrm{Ju}$ 2016).

45. National Health Pact 2014-2016 (art. 26), http://www.salute.gov.it/ imgs/C_17_pubblicazioni_2309_allegato.pdf (accessed 10 Jul 2016).

46. Law n. 208, 28 December 2015, Stability Law-Legge di stabilità 2016, http://www.gazzettaufficiale.it/eli/id/2014/12/29/14G00203/sg (accessed 10 Jul 2016).

47. Law n. 190, 23 December 2014, Stability Law-Legge di stabilità 2015, http://www.gazzettaufficiale.it/eli/id/2015/12/30/15G00222/sg (accessed 10 Jul 2016).

48. Wilde $\mathrm{MH}$, Brasch J, Zhang Y. A qualitative descriptive study of self-management issues in people with long-term intermittent urinary catheters. J Adv Nurs 2011;67:1254-63.

49. Drummond M, Griffin A, Tarricone R. Economic evaluation for devices and drugs-same or different? Value Health 2009;12:402-4.

50. http://www.eunethta.eu (accessed 10 Jul 2016).

51. Wyndaele JJ. Complications of intermittent catheterization: their prevention and treatment. Spinal Cord 2002;40:536-41.

52. Hellström $P$, Tammela $T$, Lukkarinen $O$, et al. Efficacy and safety of clean intermittent catheterization in adults. Eur Urol 1991;20:117-21.

53. Vaidyanathan S, Soni BM, Dundas S, et al. Urethral cytology in spinal cord injury patients performing intermittent catheterisation. Paraplegia 1994;32:493-500. 